\title{
Utilizing DSP to Construct Optimal Sonar Detector
}

\author{
K.H.Moustafa
}

Department of Computer Science, Misr International University, Cairo, Egypt

\section{Corresponding author: K H Moustafa [khaled.hussein@miuegypt.edu.eg]}

\section{ARTICLE DATA}

\begin{tabular}{l} 
Article history: \\
Received 05 Jan 2022 \\
Revised 08 Feb 2022 \\
Accepted 09 Feb 2022 \\
Available online \\
\hline \\
Keywords: \\
DSP (Digital Signal Processing) \\
Sonar Detector \\
Radar \\
Digital Filters \\
Matched Filter \\
S/N (Signal-to-Noise ratio) \\
Transversal Digital Filter
\end{tabular}

\begin{abstract}
The aim of this paper is to present the details of signal processing techniques in radars. These techniques are strongly based on mathematics and especially on stochastic processes. Detecting a target in a noisy environment is a many folds sequential process. Two applications of the suggested sonar detector will be discussed: the cross-correlator detector and the matched filter detector. In each scenario, we are interested in the detector's output while the input signal is free of noise and when the signal is contaminated by noise, which is considered to be Gaussian noise. Due to the reduced complexity and computation time associated with the matched filter construction, the cross-correlator detector's complexity can be solved.

Additionally, this article presents a digital implementation of a modified recursive tapped delay line integrator (transversal filter). A delay line constructed using analogue techniques has a big dimension and is unstable. Many of these issues are addressed by the digital application of this filter. Additionally, this work offers a digital implementation of a modified recursive SPMF (Single Pulse Matched Filter) and a modified recursive matched filter. Thus, an optimal sonar detector is developed and implemented using a digitally updated recursive matching filter.
\end{abstract}

\section{Introduction}

Radar, sometimes known as active sonar, is a technology that transmits a known-shaped wave and receives the echoes returned by various obstacles or targets. The transmitted wave can be of pure frequency or modified in terms of amplitude, phase, or frequency. At the point of reception, this wave must be amplified and analyzed in some way. This amplification has the effect of inflating the received signal with false noise. In general, this noise can be classified as white and Gaussian, depending on the frequency band used. If the target is stationary, the received signal is a noisy reproduction of the sent signal, with a delay equal to the target's two-way route and modified by a complex coefficient (amplitude and phase) due to attenuation caused by propagation and reflection from the target. Although the existence of this complex coefficient is unknown, signal processing must obviously take it into account [1]. If the target has a constant range-rate motion, the Doppler Effect generates further distortion of the transmitted signal by a frequency shift proportional to the radial velocity of the target and to the transmitted frequency. In general, the received signal in active radar or sonar can be modeled, to a first approximation, by a replica of the transmitted signal, which is distorted in a known manner when the set of parameters is known and multiplied by an unknown complex coefficient and to which Gaussian noise has been added [2],[10].

The improvement of a sonar system entails optimizing the following factors: [5]

- The transmitted signals (waveform, carrier frequency, transducer positioning, or, in passive systems, the frequency bands used.

- The receiver structure in order to maximize the accuracy of target parameter estimation or detection probability given the noise and spurious signal characteristics.

The effectiveness of the present-day submarine depends upon its ability to remain undetected for long periods of time while it searches, tracks, or attacks from beneath the sea surface. Before a submarine can be attacked, it must be detected.

The following processors are used to increase detection performance in this article:

- The digital SPMF is used to increase the signal-to-noise ratio of a single pulse S-time in comparison to an analogue filter, where $S$ is the number of samples contained inside the pulse. 
K.H.Moustafa et al. Journal of Computing and Communication Vol.1, No.1, PP. 65-79, 2022

- The suggested digital transversal filter improves the signal-to-noise ratio of a train of N-pulses by N-time when compared to an analogue filter, where $\mathrm{N}$ is the number of pulses inside the pulse repetition period.

- The suggested digital matched filter improves the signal-to-noise ratio of a train of N-pulses by SN times.

- A modified sonar detector based on a digital modified recursive matching filter is proposed.

All of these processors are implemented in both recursive and non-recursive fashion.

\section{Digital Filter-Based Optimization of Sonar Performance}

Take the signal transmitted by the radar or sonar's transducer as an example. Due to the fact that this signal is often narrowband, it can be expressed as:

$$
s(t)=u(t) e^{j w_{0} t}
$$

Where $u(t)$ is the complex envelope of the transmitted signal; where $s(t)$ is the carrier's angular frequency [5,13]. Then, the signal received from a target at range and with radial velocity $v$, is as follows:

$$
A u\left(t-2 R_{\mathrm{O}} / c\right) e^{-j \varphi} e^{j \omega_{0}(1+2 v / c) t}
$$

If $v / c<<1 \rightarrow B T<<c / 2 v$

Where $B$ denotes the bandwidth of the signal, $T$ is the duration of the signal, and $A$ denotes the amplitude of the received signal. Additionally, this signal is spelled as:

$$
\begin{aligned}
& \alpha u\left(t-2 R_{\mathrm{O}} / c\right) e^{j \omega_{\mathrm{O}} 2 v t / c} e^{-j \varphi} e^{j \omega_{\mathrm{O}} t} \\
& \alpha a(t, \theta) e^{-j \varphi} e^{j \omega_{\mathrm{O}} t}
\end{aligned}
$$

Where $\theta$ is the vector containing the parameters to be measured, which equals: is the vector of the parameters to be measured, equal to:

$$
\begin{aligned}
& \left(\begin{array}{c}
2 R_{\mathrm{O}} / c \\
2 \omega_{\mathrm{O}} v / c
\end{array}\right) \\
& a(t, \theta)=u\left(t-2 R_{\mathrm{O}} / c\right) e^{j \omega_{0} 2 v t / c}
\end{aligned}
$$

It is self-evident that the fundamental difficulty of sonar is to determine the arrival time and frequency of a signal. The two-way travel of the sent wave has the following consequences on the radar or sonar signal received [5].

- A delay of $2 R 0 / c$.

- A phase shift.

- A shift in the received frequency (Doppler Effect $f \mathrm{~d}=2 v / \lambda$ ).

The impulse response of a filter that is matched to a particular received signal is identical to the wave shape of the received signal. The intermediate frequency (IF) amplifier [6] is a simplified version of a matched filter that utilizes the received reflected pulse from the target. If the target's reflected echo is a train of N-pulses, integrating these pulses can significantly increase target detectability. Integration may take place in the receiver's IF section (pre detection integration) or in the video portion (post detection integration) (post detection integration). Additionally, integration can be utilized to eliminate synchronous interference. In this situation, the input signal is quantized, i.e. it is assigned one of two levels " 0 " or " 1 " based on the received signal's amplitude comparison to a threshold level. When the threshold is set too low, the likelihood of a false alert increases while the likelihood of 
K.H.Moustafa et al. Journal of Computing and Communication Vol.1, No.1, PP. 65-79, 2022

detecting the target falls. If this level is too high, the converse occurs. Typically, the threshold level is chosen using the NeymanPearson optimization criterion [6], which results in an increase in the rate of false alarms. The integrator's output is compared to a second threshold level. This level corresponds to the output if M-pulses are received [M N], so that if the integrator outputs above this level, the target is assumed to be true; if it is less than this level, the received pulses are assumed to be noise or asynchronous jamming. This is referred to as the M-out-of-N criterion [4,12].

The impulse invariance technique is a time-honored technique for creating digital filters [4]. Given a frequency characteristic, the analogue filter transfer function that best approximates is obtained using an appropriate analogue approach. In [9], the following approach is used to obtain the digital filter transfer function. Time and frequency domains are used to explain the digital filters [14].

The phrase "improvement factor" is used to characterize the filter's effect on the signal-to-noise ratio [S/N]. It has the following definition [8]:

$$
I=\frac{(S / N)_{\text {output }}}{(S / N)_{\text {input }}}
$$

Where $(S / N)=\frac{\text { peak signal power }}{\text { average noise power }}$

Assume the input signal is a series of rectangular pulses of amplitude A and the noise is Gaussian in distribution with a standard deviation of $\sigma$.

\subsection{Filter with a single matched pulse}

The impulse response of a matched filter is defined as follows [9]:

$$
h(t)=X^{*} \cdot\left(t_{o p}-t\right)
$$

Where $t_{o p}$ is an optimal delay that causes the filter to be causal, and (*) indicates the complex conjugate with (). For post detection processing $X(t)$, the image of the input signal $h(t)$ is used. Due to the rectangular pulse $X(t)$ having the following definition:

$$
X(t)=\left\{\begin{array}{cc}
1 & 0 \leq t \leq t_{P} \\
0 & O . W
\end{array}\right.
$$

The Filter with a single matched pulse is found to have the following impulse response:

$$
h(t)=\left\{\begin{array}{cc}
1 & 0 \leq t \leq t_{P} \\
0 & O . W
\end{array}\right.
$$

The analog implementation of equation (11) is shown in Figure 1

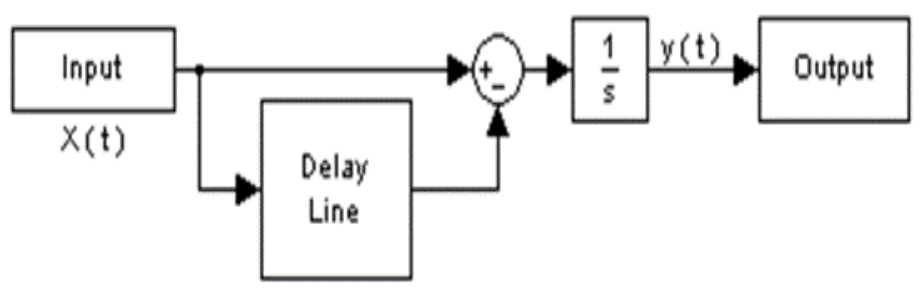

Figure 1: Analog implementation of a filter with a single matched 
K.H.Moustafa et al. Journal of Computing and Communication Vol.1, No.1, PP. 65-79, 2022

The filter's output is the autocorrelation function, which takes the form [4] in our case.

$$
y(t)=\left\{\begin{array}{cc}
t & 0 \leq t \leq t_{p} \\
2 t_{p}-t t_{p} \leq t \leq 2 t_{p} & \\
0 & O . W
\end{array}\right.
$$

Figure 2 illustrates the impulse response of a filter with a single matched pulse

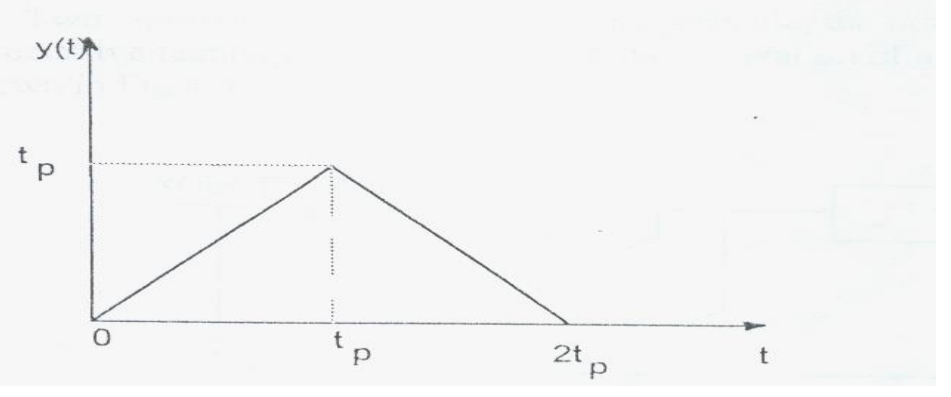

Figure 2: Filter impulse response to a single matched pulse

If $f_{S}$ is the sampling frequency used to implement this filter digitally, then there are S samples during the pulse duration, where $\mathrm{S}$ is defined as:

$$
S=t_{P} \cdot f_{S}
$$

The impulse response that corresponds to this is as follows:

$$
h\left(n T_{S}\right)=\left\{\begin{array}{cc}
1 & n=0,1, \ldots, s-1 \\
0 & o . w .
\end{array}\right.
$$

Then, the Z-domain representation of this filter, $H(Z)$, is given by [4]:

$$
H(Z)=1+Z^{-1}+Z^{-2}+\ldots .+Z^{-(S-1)}
$$

The non-recursive filter's frequency response to a single matched pulse is given by [4]:

$$
H\left(e^{-j W}\right)=1+e^{-j W T_{S}}+e^{-2 j W T_{S}}+\ldots .+e^{-j(S-1) W T_{S}}
$$

Additionally, the time domain response of this filter is specified by:

$$
y\left(n T_{S}\right)=X\left(n T_{S}\right)+X\left((n-1) T_{S}\right)+X\left((n-2) T_{S}\right)+\ldots .+X\left((n-(s-1)) T_{S}\right)
$$

Figure 3 illustrates a non-recursive implementation of a digital filter using a single matched pulse with $S=10$

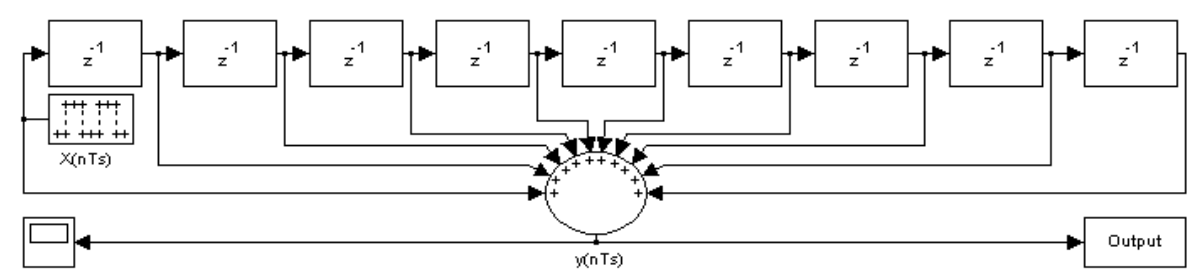

Figure 3: Realization of non-recursive digital filter with a single matched pulse for $S=10$. 
Figure 4 and Figure 5 illustrate the temporal and frequency domain responses of a non-recursive filter with a single matched pulse for $S=10$ and 20 .

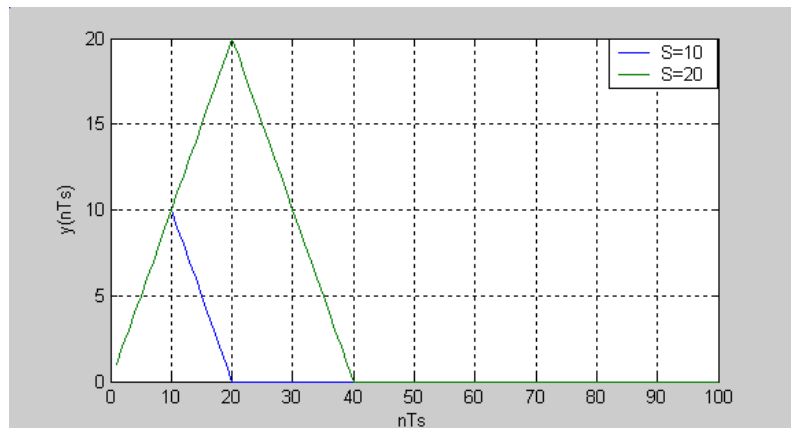

Figure 4: Time domain response of non-recursive filter with a single matched pulse for $S=10, S=20$

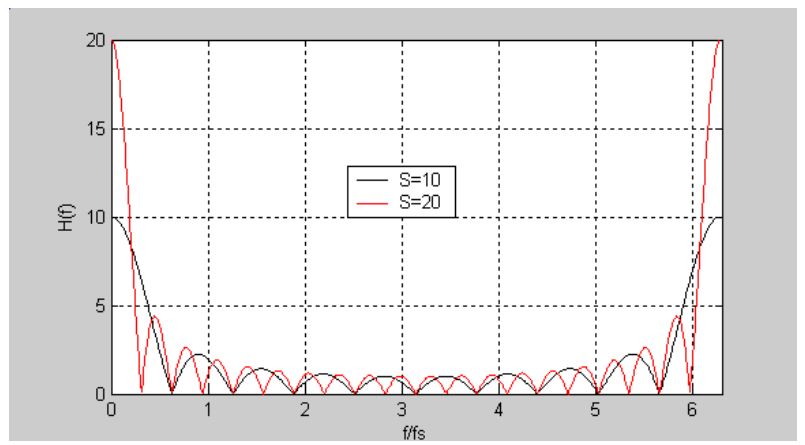

Figure 5: Frequency domain response of non-recursive filter with a single matched pulse for $S=10, S=20$

It is obvious that when S increases, the autocorrelation function's peak increases. With a single matched pulse, the non-recursive digital filter is always stable. Meanwhile, it suffers from a slow processing speed and a high degree of complexity in its realization. The digital filter with a single matched pulse is used to enhance the receiver's performance when only one received pulse is received. The enhancement is proportional to the number of samples contained inside the pulses. This value is dependent on the duration of the pulse and the sampling period. The number of samples within one pulse is ten in the case of $[\delta=100 \mathrm{~ms}$, Ts $=10 \mathrm{~ms}]$. $[9,12]$ express the transfer function of a recursive digital filter with a single matched pulse as follows:

$$
H(Z)=\left(1-Z^{-S}\right) /\left(1-Z^{-1}\right)
$$

Figure 6 depicts the corresponding implementation.

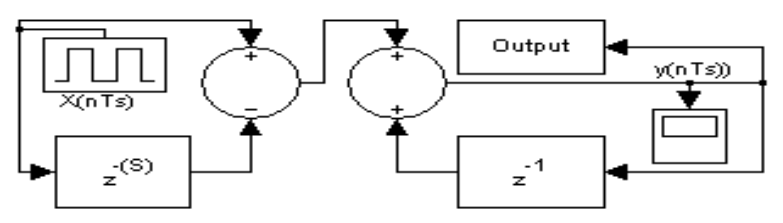

Figure 6: Recursive realization of digital filter with a single matched pulse

The problem of using a single matched pulse to realize a recursive digital filter is that it is only partially stable. Stabilization of such a filter is accomplished by multiplying a feedback recursive branch by a stabilization coefficient A. The stabilized function is then defined as follows:

$$
H(Z)=\left(1-Z^{-S}\right) / A \cdot\left(1-Z^{-1}\right)
$$

Figure 7 illustrates the implementation of a stabilized recursive digital filter using a single matched pulse. 


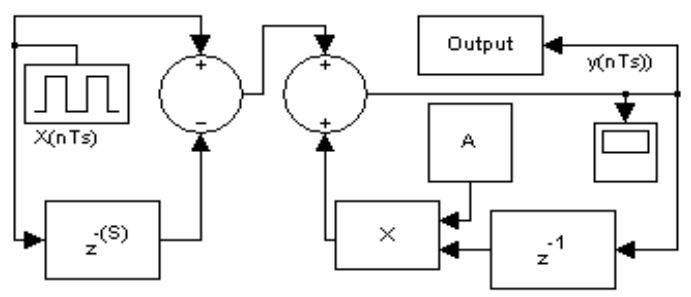

Figure 7: Stabilized recursive digital filter with a single matched pulse

The time and frequency domain responses of a stabilized recursive digital filter are determined for various stabilization coefficients using a single matched pulse. The temporal and frequency domain responses of the stabilized recursive digital filter with a single matched pulse are shown in Figures 8 and 9, respectively, for $S=10$.

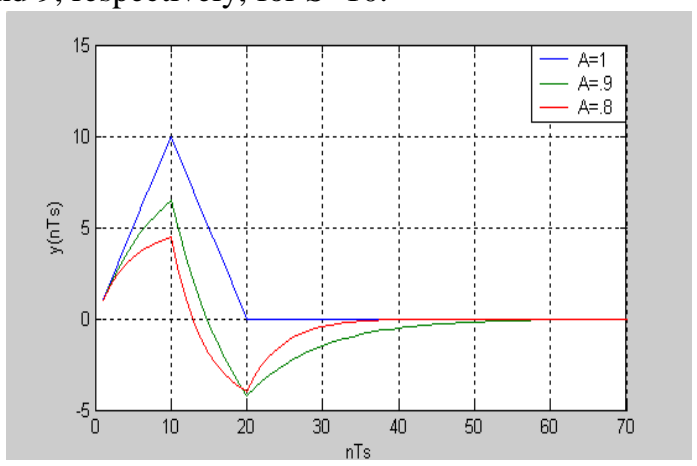

Figure 8 Time domain response of the stabilized recursive digital filter with a single matched pulse for $S=10$, and $\mathrm{A}=1.0,0.9$, and 0.8

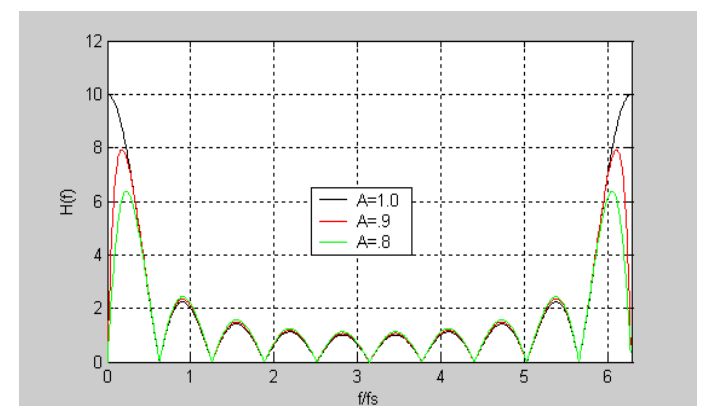

Figure 9: Frequency response of the stabilized recursive digital filter with a single matched pulse for $\mathrm{S}=10$, and $\mathrm{A}=$ $1.0,0.9$, and 0.8

It is obvious that multiplying by the stabilizing coefficient A affects the space of the filter's output slightly. This distortion becomes more pronounced as the multiplication coefficient's value lowers. This distortion can be eliminated by adding another multiplication factor. Then equation (18) is more likely to:

$H(Z)=\frac{1-\left(A Z^{-1}\right)^{S}}{1-A Z^{-1}}$

Because the factor is one of the numerator factors, it may be calculated using the denominator's one zero. Figure 10 illustrates the implementation of a modified recursive digital filter with a single matched pulse.

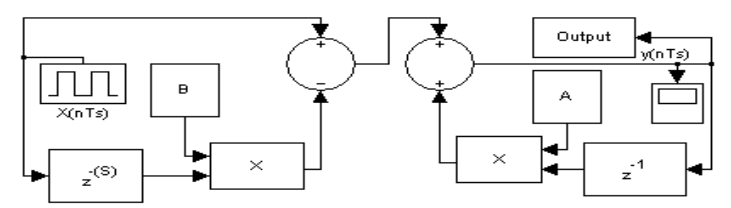

Figure 10: Modified recursive digital filter with a single matched pulse

Figure 11 and Figure 12 illustrate the temporal and frequency domain responses of the improved recursive digital filter with a single matched pulse for various multiplication factors. 


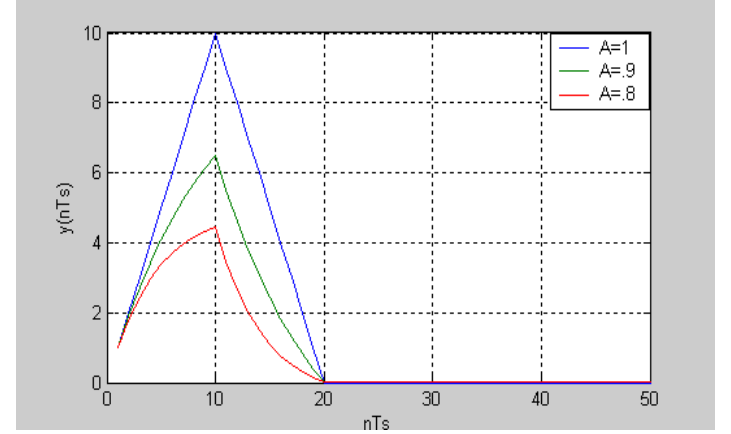

Figure 11: Time domain response of the modified recursive filter with a single matched pulse for $S=10$, and $A=1.0,0.9$, and 0.8

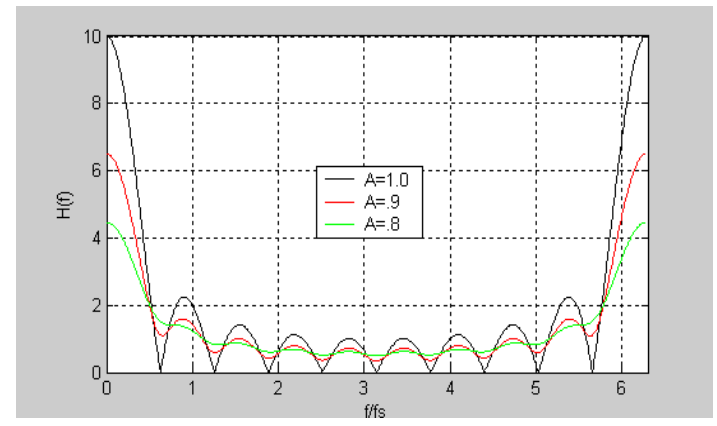

Figure 12: Frequency response of the modified recursive filter with a single matched pulse for $S=10$, and $A=1.0,0.9$, and 0.8

The $(\mathrm{S} / \mathrm{N})$ ratio of a filter with a single matched pulse whose sample count is $\mathrm{S}$, is given by:

$$
(S / N)_{\text {input }}=\frac{A^{2}}{\sigma^{2}}
$$

This filter's peak output is determined to be:

$$
y(n)_{\text {peak }}=S A
$$

Figure 15 Time domain response of a non-recursive transversal digital filter for $\mathrm{N}=10, \mathrm{~L}=100$ and the average output noise power, $\overline{m_{0}^{2}}$ of the filter is given by:

$$
\overline{m_{0}^{2}}=S \sigma^{2}
$$

Finally, the filter's output can be expressed as:

$$
(S / N)_{\text {output }}=(A S) /\left(S \sigma^{2}\right)
$$

Then, the enhancement factor of a digital filter with a single matched pulse is calculated as follows:

$$
I=S
$$

\subsection{Transversal Digital Filter}

The processing of an $\mathrm{N}$-video pulse train necessitates the use of a transversal digital filter. A transversal digital filter's analogue implementation requires a tapped delay line with $(\mathrm{N}-1)$ sections, each with a delay period of Figure 13 illustrates the implementation of a non-recursive transversal digital filter.

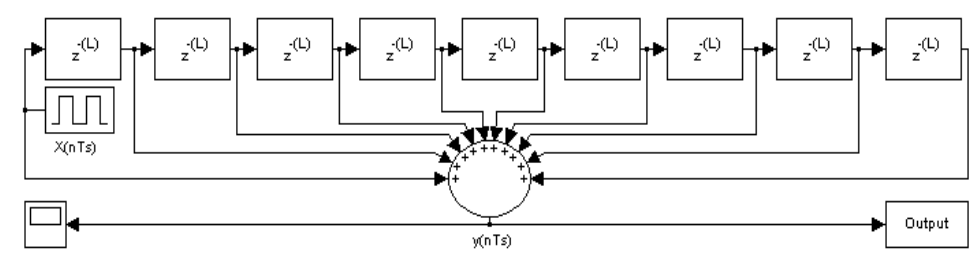

Figure 13: Implementation of the transversal digital filter in a non-recursive fashion for $\mathrm{N}=10$ 
K.H.Moustafa et al. Journal of Computing and Communication Vol.1, No.1, PP. 65-79, 2022

The time domain response of the non-recursive transversal digital filter for $\mathrm{L}=2$, the pulse repetition time, and $\mathrm{N}=10$ is shown in Figure 14 while its frequency response is shown in Figure 15.

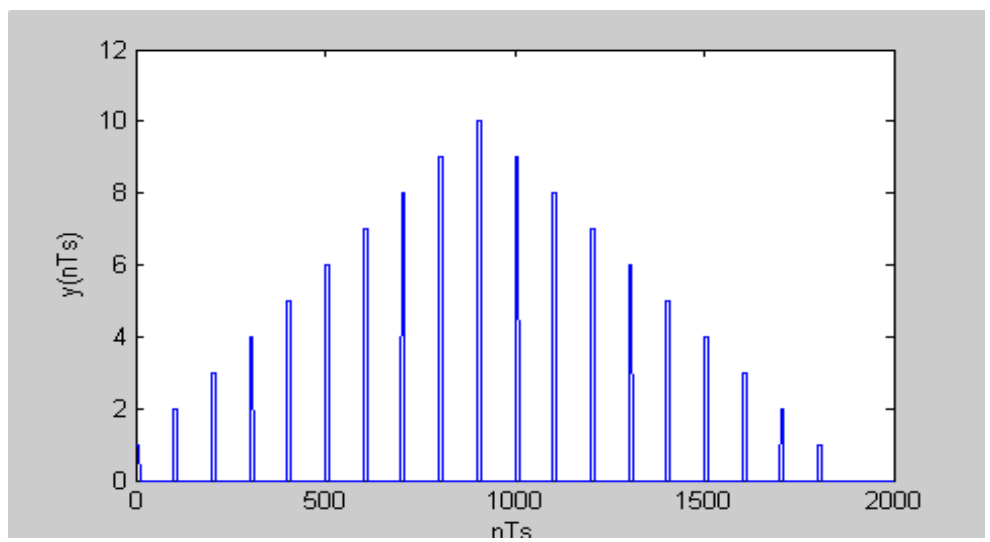

Figure 14: Time response of a non-recursive transversal digital filter for $\mathrm{N}=10$, and $\mathrm{L}=2$

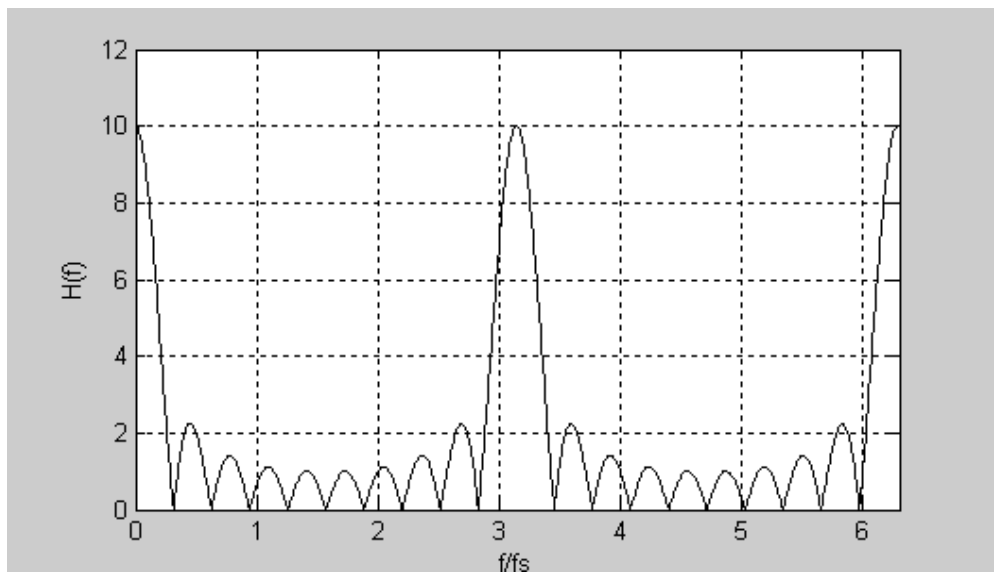

Figure 15: Frequency response of a non-recursive transversal digital filter for $\mathrm{N}=10$, and $\mathrm{L}=2$

Similarly to the situation of a digital filter with a single matched pulse, the modified transfer function of a transversal digital recursive filter is as follows:

$$
H(Z)=\frac{1-\left(A Z^{-L}\right)^{N}}{1-A Z^{-L}}
$$

The realization of a modified recursive transversal digital filter is shown in Figure 16

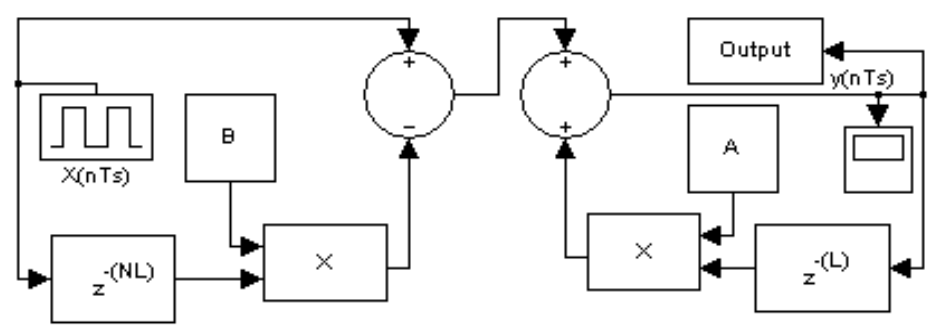

Figure 16: Realization of a modified recursive digital transversal filter

Figure 17 illustrates the time domain response of a modified recursive transversal digital filter with $\mathrm{L}=90, \mathrm{~N}=10$, and $\mathrm{A}=0.95$. Figure 18 illustrates the frequency response of a modified recursive transversal digital filter for $\mathrm{L}=2, \mathrm{~N}=10, \mathrm{~A}=0.98,0.90,0.80$. 


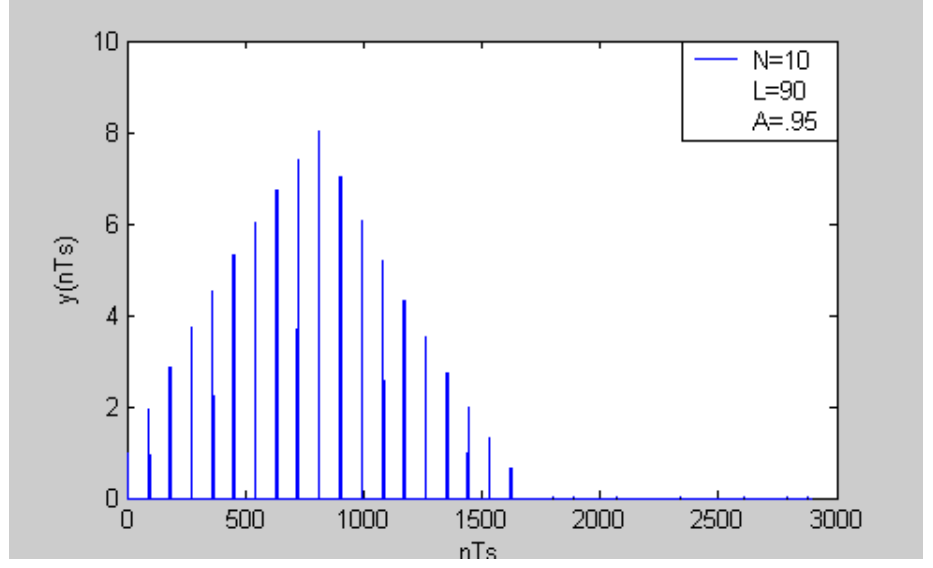

Figure 17: Time domain analysis of a modified recursive transversal digital filter for $\mathrm{N}=10$, $\mathrm{L}=100$ and $\mathrm{S}=10$

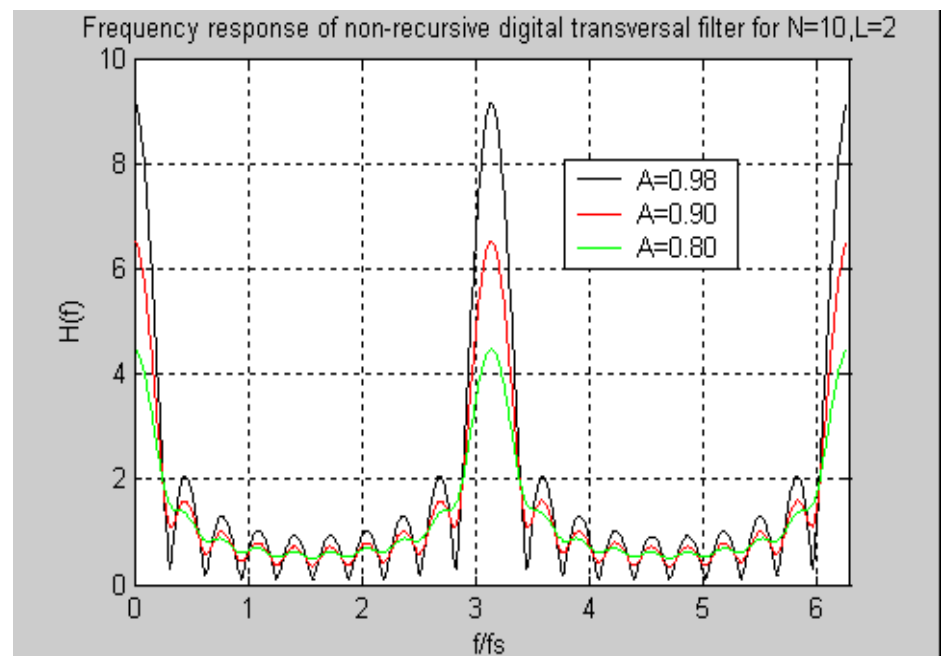

Figure 18: Frequency response of a modified recursive transversal digital filter for $\mathrm{L}=2, \mathrm{~N}=10, \mathrm{~A}=0.98,0.90,0.80$

This filter's maximum output is calculated to be:

$$
y(n)_{\text {peak }}=N A
$$

The filter's average output noise power $\overline{m_{0}^{2}}$ is determined by:

$$
\overline{m_{0}^{2}}=N \sigma^{2}
$$

The filter's output $(S / N)$ is denoted by the formula:

$$
(S / N)_{\text {output }}=\left(A N^{2}\right) /\left(N \sigma^{2}\right)
$$

Binary integration's enhancement factor is given by:

$$
\boldsymbol{I}=N
$$

\subsection{Filter matched digitally}

The digital matched filter for groups of $\mathrm{N}$-video pulses is composed of a transversal digital filter for N-pulse integration cascaded with a single matched pulse filter. Before or after the transversal digital filter, the filter with a single matched pulse can be utilized [4]. Figure 19 illustrates a non-recursive implementation of a digital matched filter with $\mathrm{S}=10$ and $\mathrm{N}=10$. 
K.H.Moustafa et al. Journal of Computing and Communication Vol.1, No.1, PP. 65-79, 2022

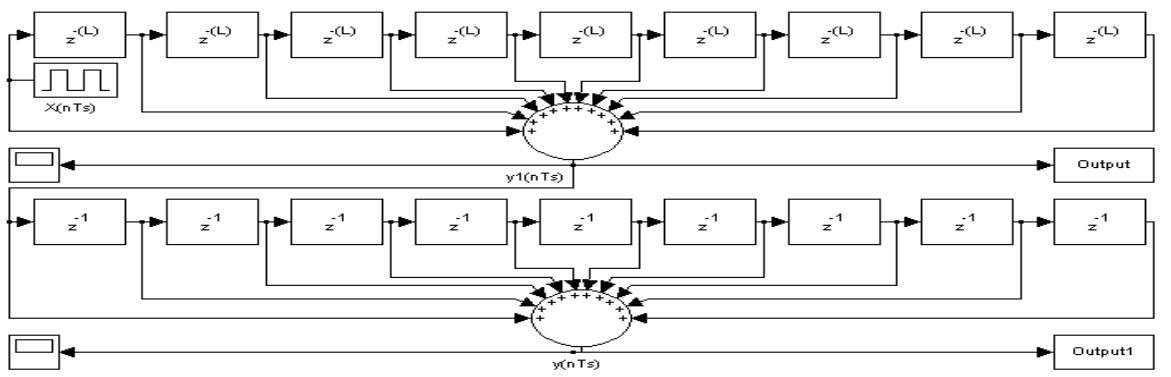

Figure 19: Non-recursive realization of a digital matched filter for $S=10$ and $N=10$

The time and frequency domain response of a modified recursive digital matched filter for $\mathrm{N}=10, \mathrm{~S}=10$, and $\mathrm{L}=40$ are shown in Figures 20 and 21.

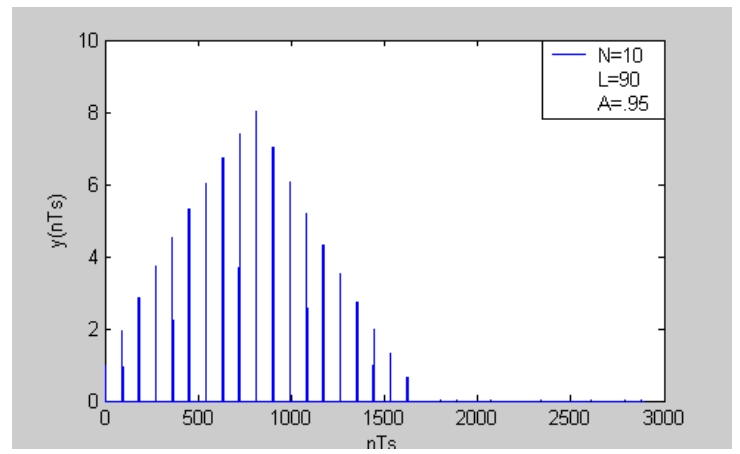

Figures 20: Time domain response of a modified recursive digital matched filter for $\mathrm{N}=10, \mathrm{~L}=40$ and $\mathrm{S}=10$

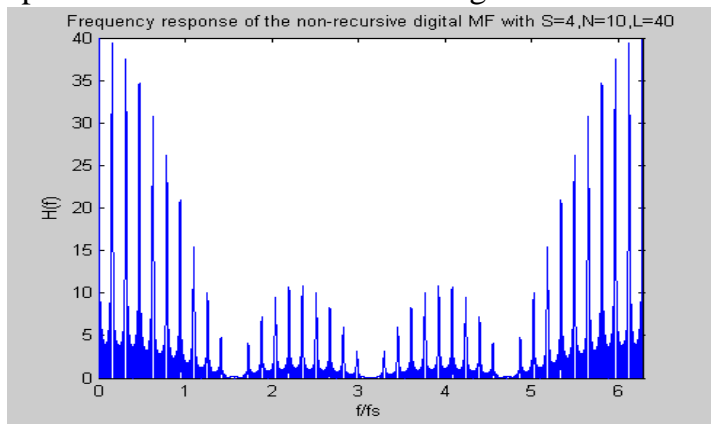

Figures 21: Frequency response of a modified recursive digital matched filter for $\mathrm{N}=10, \mathrm{~L}=40$ and $\mathrm{S}=10$

The digital matching filter produces the following peak output:

$$
y(n)_{\text {peak }}=(N S) \cdot A
$$

The filter's average output noise power $\overline{m_{0}^{2}}$ is determined by:

$$
\overline{m_{0}^{2}}=N S \sigma^{2}
$$

The filter's output $(S / N)$ is as follows:

$$
(S / N)_{\text {output }}=(A N S)^{2} /\left(N S \sigma^{2}\right)
$$

Finally, the enhancement factor of the improved recursive digital matching filter can be calculated as follows:

$$
I=N S
$$

\section{Development of an optimal sonar detector}

The challenge of signal $s(t)$ detection in noise $n(t)$ entails deciding between two hypotheses based on the received signal $r(t)$ :

$$
\begin{aligned}
& H_{1}: r(t)=s(t)+n(t) \\
& H_{0}: r(t)=n(t)
\end{aligned}
$$


Numerous criteria can be used; the most natural, and closest to the technique used in practice, is to examine two distinct types of error:

- Deciding that there is a target $\left(H_{1}\right)$ when only noise is present $\left(H_{0}\right)$ : this is a false alarm, with probability $P_{f a}$ for a given decision rule;

- Deciding that there is no target $\left(H_{0}\right)$ when hypothesis $H_{1}$ is in fact true: this is a non-detection of probability $P_{n d}$.

It is self-evident that the two faults are mutually exclusive in practice. By reducing the likelihood of one of these errors occurring, you automatically increase the likelihood of the other occurring. The Neyman-Pearson practical criterion is utilized, which entails setting the false alarm probability to a predefined number and minimizing the non-detection probability [5]. The NeymanPearson criterion is used as an optimization criterion in order to optimize detection probability while maintaining a constant false alarm probability. Typically, targets are recognized against a white Gaussian noise backdrop.

The optimal detector is one that is based on this criterion. The structure of an ideal detector is depicted in Figure 22 (a). This detector is composed of the following steps: correlation with a replica of the expected signal (for each conceivable parameter), quadrature detection, normalization to the replica power, and comparison of these values to a threshold determined according to the required false alarm probability [5], [11].

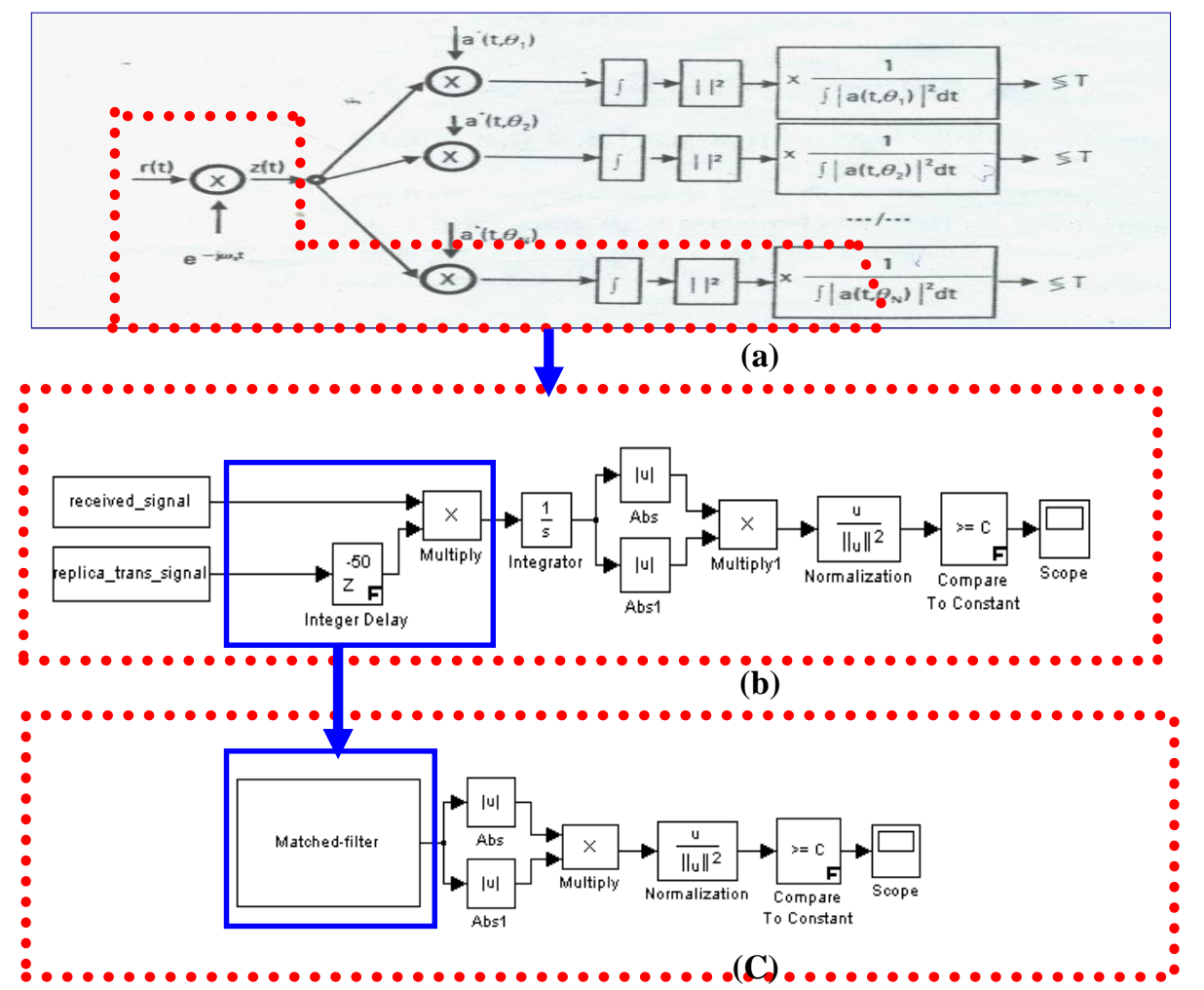

Figure 22: The ideal sonar detector's structure

This article discusses two distinct types of sonar detectors. The first model is a cross-correlator detector as illustrated in Figure 22 (b), and the second model is a modified recursive digital matched filter detector as illustrated in Figure 22 (c). In each scenario, we are interested in the detector's output for two cases; the first case is the noise free input signal, and the second one while the input signal is surrounded by Gaussian noise.

A set of channels corresponding to the estimated time delays from the echo target is required by the cross-correlator detector. There is a separate channel for each time delay, which increases implementation complexity. Rather than multiplying the input signal by a stored replica of the transmission signal, the matched filter detector multiplies the received signal by itself delayed in time. Due to its simplicity and speed of calculation, the cross-correlator detector's difficulty can be solved using a matched filter. As a result, our research focuses exclusively on one channel of an optimal detector. This channel corresponds to the goal echo delay of 50 milliseconds. As illustrated in Figure 22, this channel is enclosed by a dashed line (a). The input for two sonar detector models reflects a practical sonar system [3], which is considered to be a train of four rectangular pulses $(\mathrm{N}=4)$, each having a pulse width of 10 milliseconds $(S=10)$, a pulse repetition period of $\mathrm{L}=100$ milliseconds, and ten samples within each pulse. 
K.H.Moustafa et al. Journal of Computing and Communication Vol.1, No.1, PP. 65-79, 2022

\subsection{Development of a sonar detector with cross-correlation}

\subsubsection{Consider the case of a noise-free signal.}

The cross-correlator detector's input and output are depicted in Figures 23 and 24.

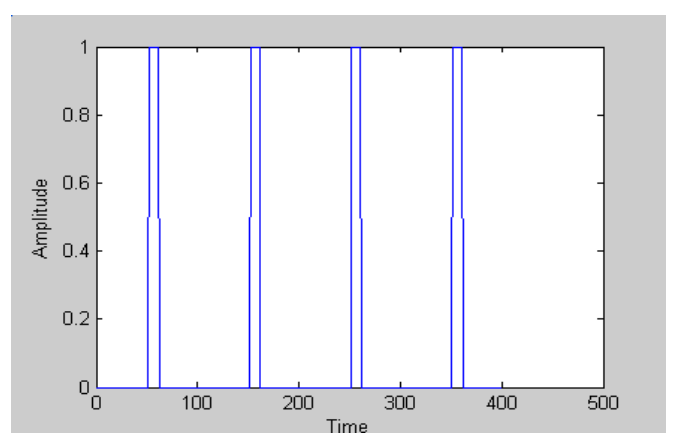

Figure 23: Cross-correlation input with $\mathrm{N}=4, \mathrm{~S}=10 \mathrm{~ms}$, and $\mathrm{L}=100 \mathrm{~ms}$

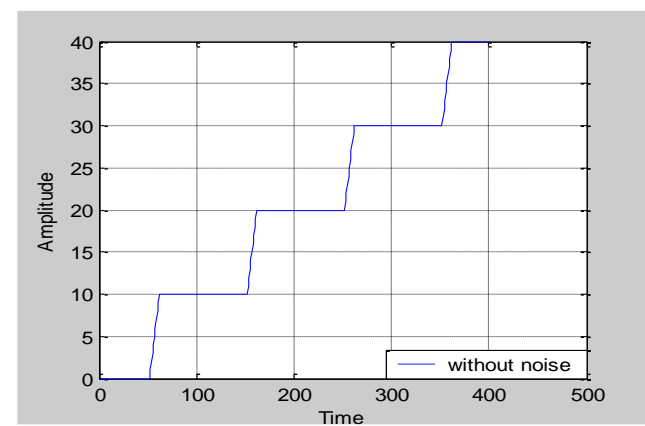

Figure 24: Cross-correlation output for $\mathrm{N}=4, \mathrm{~S}=10 \mathrm{~ms}$, and $\mathrm{L}=100 \mathrm{~ms}$

\subsubsection{Consider the case of a signal that has been corrupted by Gaussian noise}

As illustrated in Figure 25, the cross-correlator detector is activated by a signal plus Gaussian noise, while the equivalent output is illustrated in Figure 26.

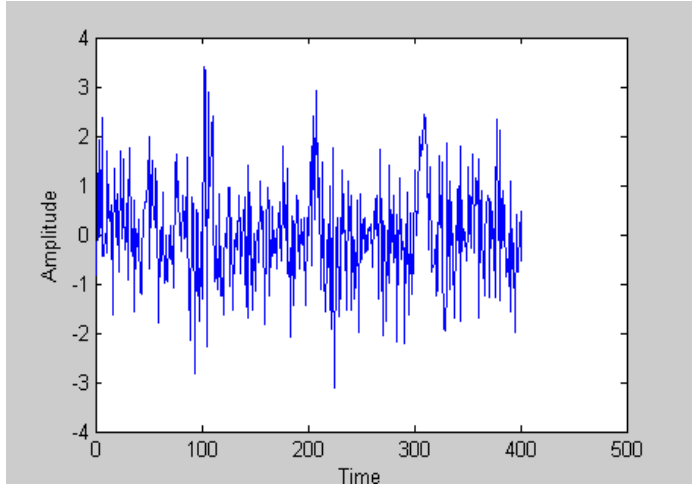

Figure 25: Cross-correlation input with $\mathrm{N}=4, \mathrm{~S}=10 \mathrm{~ms}$, and $\mathrm{L}=100 \mathrm{~ms}$ and contaminated by Gaussian noise 


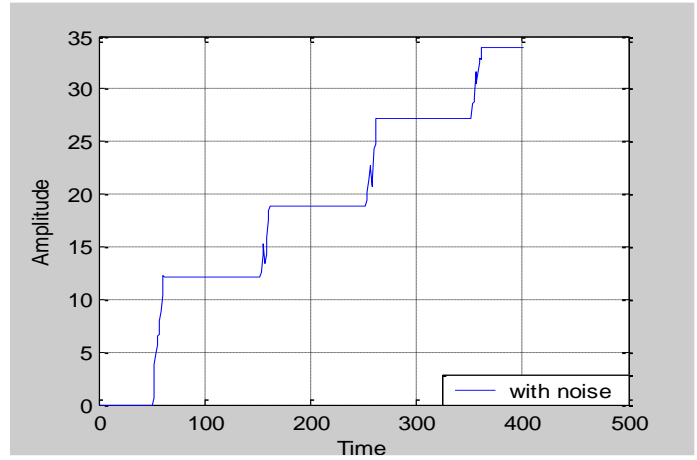

Figure 26: Cross-correlation output contaminated by Gaussian noise for an input of $\mathrm{N}=4, \mathrm{~S}=10 \mathrm{~ms}$, and $\mathrm{L}=100 \mathrm{~ms}$.

\section{Realization of a modified recursive digital matched filter sonar detector}

The digital matching filter performs an autocorrelation function between the received signal and a frequency response replica of the transmitted signal. However, while the cross correlation function is technically equal to the autocorrelation function, they are implemented differently in practice [7]. This indicates that the above-mentioned cross-correlator detector can be substituted with a modified recursive digital matching filter. Additionally, this digital filter is activated by a train of four rectangular pulses $(\mathrm{N}=4)$, each having a pulse width of 10 milliseconds $(S=10)$, a pulse repetition period of $\mathrm{L}=100$ milliseconds, and ten samples within each pulse.

\subsection{Consider the case of a noise-free signal.}

The digital matched filter detector's input and output are depicted in Figures 27 and 28.

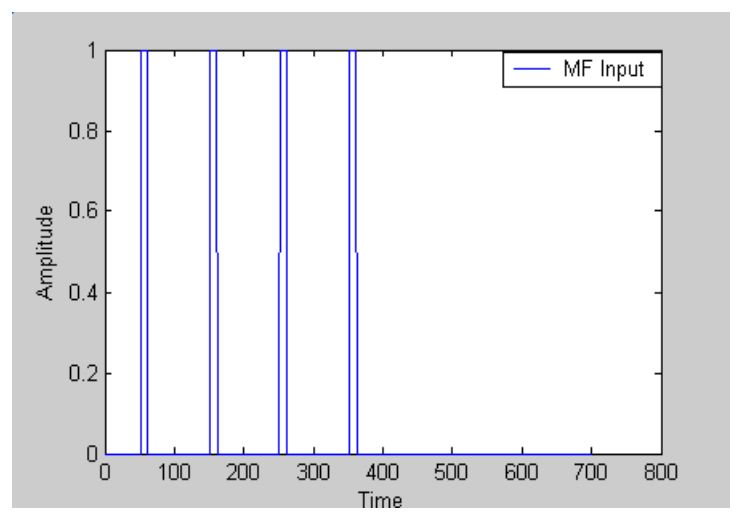

Figure 27: The input to the digital matched filter has the following parameters: $\mathrm{N}=4, \mathrm{~S}=10 \mathrm{~ms}$, and $\mathrm{L}=100 \mathrm{~ms}$

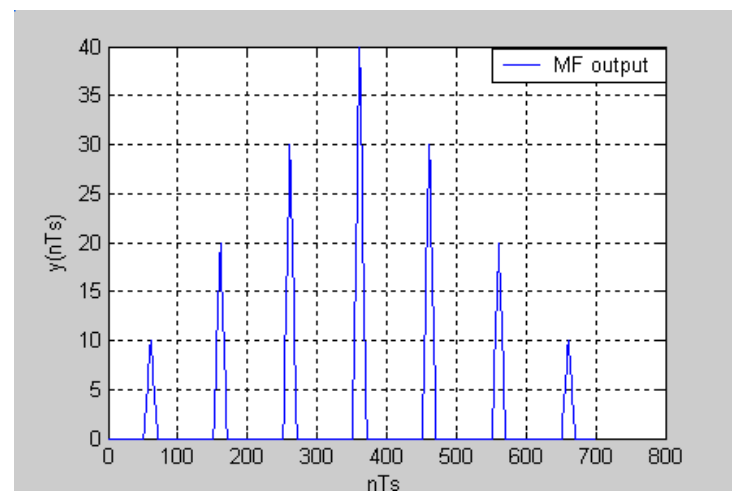

Figure 28: The output of the digital matched filter when $\mathrm{N}=4, \mathrm{~S}=10 \mathrm{~ms}$, and $\mathrm{L}=100 \mathrm{~ms}$ are used as inputs.

\subsection{Consider the case of a signal that has been corrupted by Gaussian noise}

As illustrated in Figure 29, the digital matched filter detector is also activated by a signal plus Gaussian noise, and the associated output is displayed in Figure 30. 


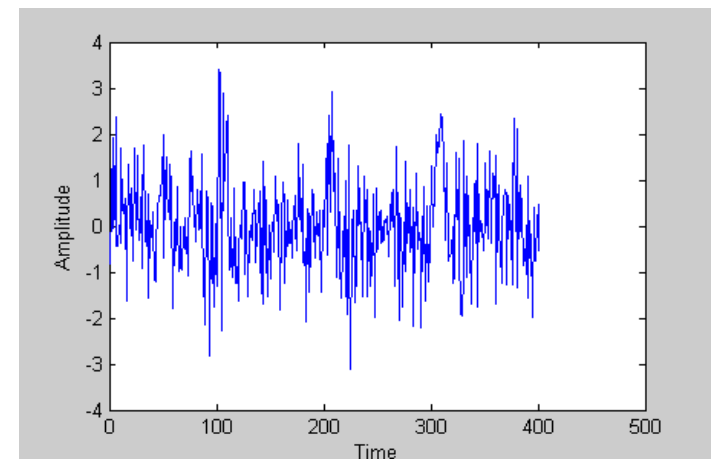

Figure 29: Input to the digital matched filter with $\mathrm{N}=4, \mathrm{~S}=10 \mathrm{~ms}, \mathrm{~L}=100 \mathrm{~ms}$, and contaminated by Gaussian noise

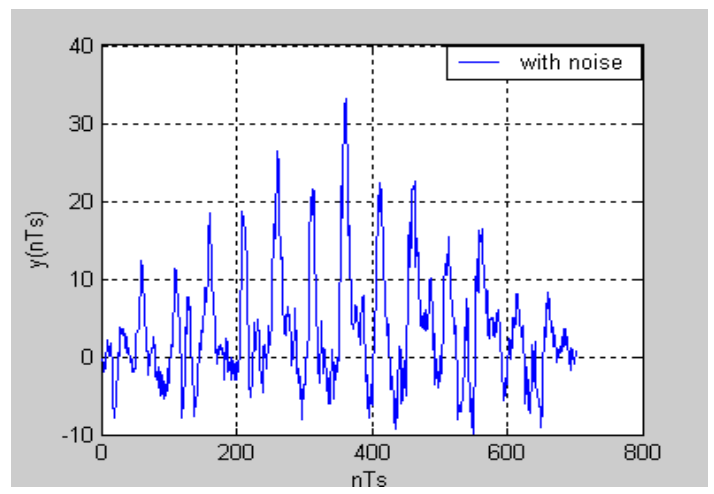

Figure 30: Output of the digital matched filter for an input of $\mathrm{N}=4, \mathrm{~S}=10 \mathrm{~ms}$, and $\mathrm{L}=100 \mathrm{~ms}$ contaminated by Gaussian noise

To verify the suggested modified sonar matched filter detector's performance in the presence of Gaussian noise, Figure 31 shows a comparison of the sonar detector's performance in the absence and presence of Gaussian noise.

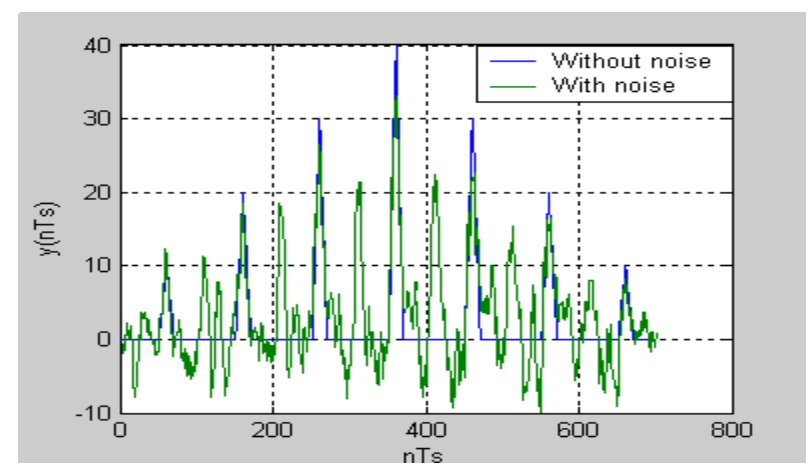

Figure 31: Comparing the outputs of a modified recursive digital matching filter for $\mathrm{N}=4, \mathrm{~S}=10 \mathrm{~ms}$, and $\mathrm{L}=100 \mathrm{~ms}$ in the presence and absence of Gaussian noise

According to the results reported previously, the cross-correlator detector performs identically to the digital matched filter detector when the signal is noise-free. A set of channels equal to the amount of time delays expected from the echo target is required for the cross-correlator. There is a separate channel for each time delay, which increases implementation complexity. Rather than multiplying the input signal by a stored replica of the sent signal, the matched filter multiplies the received signal by itself after it has been delayed in time. A digital matching filter can be used to solve the complexity of the cross-correlator structure. Finally, it is evident that the modified recursive digital matching filter is the preferable structure for the sonar detector.

\section{Conclusion}

The matching filter is a technique that is frequently employed in the identification of underwater targets using ultrasonic waves. The matched filter theory is based on knowledge of the precise shape of the pulse to be detected, and the filter is designed to maximize the SNR between the received signal and its delayed copy. Apart from the attractive attribute of boosting the SNR of its output when a signal is discovered, the matched filter is also optimal in terms of likelihood of detection. 
K.H.Moustafa et al. Journal of Computing and Communication Vol.1, No.1, PP. 65-79, 2022

Enhancing underwater detection performance requires boosting sonar detectability, which can be accomplished by utilizing either a digital single pulse matching filter or a digital delay line integrator (transversal filter), or by cascading both.

\section{References}

[1] A. D. Waite, "Sonar for practicing engineering", Ashley Waite 2002.

[2] Francois Le Chevalier, "Principles of radar and sonar signal processing", 2002.

[3] R.Frieden, "Principles of Naval Weapons systems", United States Naval Institute Press, 1985.

[4] B. A. Shenoi, "Introduction to DSP and filter design", 2006.

[5] "Sonar Modeling Handbook, Volume 1. Modeling Procedures and Environments" .DRA/ Mar TM (USSF) 91172 (original version Jan. 1992, most recent update Dec. 1994), UK Defence Research Agency.

[6] Difranco, J.V., "Radar Detection", Artech House, 1980.

[7] Skolnk, "Introduction to radar system." 2001.

[8] Paul A. Lynn, "Radar Systems", Macmillan education Ltd., 1987.

[9] A. Antoniou. "Digital filters, Analysis and Design", 1979.

[10] Martinez-Cantin, R. BayesOpt: A Bayesian Optimization Library for Nonlinear Optimization, Experimental Design and Bandits. J. Mach. Learn. Res. 2014, 15, 3735-3739.

[11] Redmon, J.; Divvala, S.; Girshick, R.; Farhadi, A. You Only Look Once: Unified, Real-Time Object Detection. In Proceedings of the 2016 IEEE Conference on Computer Vision and Pattern Recognition (CVPR), Las Vegas, NV, USA, 27-30 June 2016; pp. 779-788.

[12] D. Likhith Reddy, S.V. Subba Rao; “A Novel Design of Matched Filter for Digital Receivers “International Journal of Recent Technology and Engineering (IJRTE) ISSN: 2277-3878, Volume-8 Issue-3, September 2019.

[13] S. A. Elgamel and J. J. Soraghan, "Enhanced Monopulse Radar Tracking Using Filtering In Fractional Fourier Domain," in IEEE International Radar Conference Washington DC, USA, 2010.

[14] D. Likhith Reddy , S.V. Subba Rao," A Novel Design of Matched Filter for Digital Receivers “ International Journal of Recent Technology and Engineering (IJRTE) ISSN: 2277-3878, Volume-8 Issue-3, September 2019. 